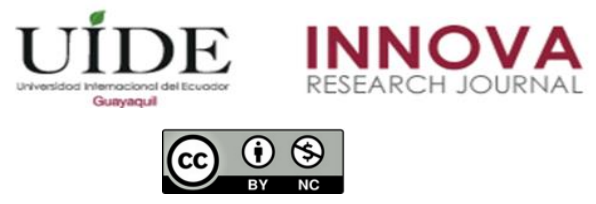

INNOVA Research Journal, ISSN 2477-9024

(Septiembre-Diciembre 2020). Vol. 5, No.3.1 pp. 244-262

DOI: https://doi.org/10.33890/innova.v5.n3.1.2020.1510

URL: http://revistas.uide.edu.ec/index.php/innova/index

Correo: innova@uide.edu.ec

\title{
Estudio sobre la tasa de interés de referencia de Estados Unidos y su comportamiento en la inversión peruana periodo 2016 al 2019
}

\section{Study on the reference interest rate of the United States and conduct in Peruvian investment period 2016 al 2019}

Lauralinda Leonor Cavero Egúsquiza Vargas

(D) https://orcid.org/0000-0001-5222-8865

Manuel Alberto Munsibay Muñoa

(D) https://orcid.org/0000-0001-5222-8865

Universidad César Vallejo, Perú

Autor para correspondencia: lauralinda.cavero@gmail.com; mmunsibay@gmail.com

Fecha de recepción: 24 de agosto de 2020 - Fecha de aceptación: 23 de noviembre de 2020

\section{Resumen}

El presente artículo, titulado Estudio sobre la tasa de interés de referencia de Estados Unidos y su comportamiento en la inversión peruana periodo 2016 al 2019, surge por la inquietud de determinar en qué medida incide la tasa de interés de referencia de Estados Unidos ante una variación de esta en las inversiones en la economía peruana hasta el año 2019. El enfoque usado fue el cuantitativo, el tipo de investigación aplicada y el diseño no experimental. El instrumento usado fue el análisis de contenido cuantitativo, para establecer la incidencia que existe entre las variables se aplica el modelo econométrico a través de la regresión lineal simple mediante el programa econométrico E-views, el cual da como resultado el coeficiente de determinación de $\mathrm{R}_{2}$ (0.47) lo que significa que la tasa interés de referencia de Estados Unidos incide en la inversión de la economía peruana en $47 \%$, concluyendo que ante una variación de $1 \%$ de la tasa de interés de referencia, esta incide $36 \%$ para las inversiones en la economía peruana, afectando a la demanda interna, movimiento de capital y al problema del endeudamiento.

Palabras claves: Tasa de interés de referencia; inversión; política monetaria; fiscal y banco centrales.

\footnotetext{
Abstract

This article, entitled Study on the reference interest rate in the United States and its behavior in Peruvian investment from 2016 to 2019, arises from the concern to determine to what extent the reference interest rate in the United States affects a variation of this in investments in the Peruvian economy until 2019, The approach used was the quantitative, the type of applied research and the non-experimental design. The instrument used was the quantitative content analysis, to establish the incidence that exists between the variables, the econometric model is applied through simple linear regression through the E-views econometric program, which results in the coefficient of determination of R2 ( 0.47 ) which means that the reference interest rate in the United States affects investment in the Peruvian economy by $47 \%$, concluding that a variation of $1 \%$ in the reference
} 
interest rate, it affects $36 \%$ for investments in the Peruvian economy, affecting domestic demand, capital movement and the debt problem.

Keywords: reference interest rate; investment; monetary policy; fiscal and central bank.

\section{Introducción}

En el Perú, subir la tasa de interés de referencia genera un problema en las inversiones que se complican a la hora de importar, porque el dólar se fortalece más, y los precios de la materia prima se incrementan en los mercados internacionales, por consiguiente el exportador no podrá cumplir la actividad económica que tiene planificada reduciendo la demanda y oferta interna, el costo de los créditos fueron significativamente altos a causa del encarecimiento de las tasas de interés de referencia, disminuyendo la inversión privada en $22 \%$ del PBI para el año 2017, esto hizo que los proyectos de inversión sean menos atrayentes al inversionista subiendo el riesgo país, provocando presiones cambiarias a corto plazo creando una incertidumbre en el desarrollo de la economía, y la volatilidad del mercado financiero en 2018 por la política monetaria de Estados Unidos, hizo que las tenciones comerciales propicien la reasignación desordenada del capital. (Banco mundial, 2018).

Por lo dicho anteriormente se plantea como problema general: ¿En qué medida incide la tasa de interés de referencia de Estados Unidos en las inversiones en la economía peruana?. Para realizar un mejor análisis se propone los problemas específicos: ¿En qué medida la tasa de interés activa incide en el nivel de demanda agregada?, ¿En qué medida las operaciones de mercado abierto inciden en el nivel de riesgo?, ¿En qué medida el precio de las operaciones crediticias incide en el nivel de rentabilidad?

Después de establecer la problemática, se propone el objetivo general para la presente investigación: Determinar en qué medida incide la tasa de interés de referencia de Estados Unidos en las inversiones en la economía peruana.

La finalidad de este trabajo es brindar conocimiento de la aplicación de la tasa de interés de referencia de Estados Unidos como instrumento de política monetaria y su influencia en el comportamiento de la inversión peruana.

\section{Marco teórico}

Para desarrollar la presente investigación se tomó como base trabajos internacionales que sustentan el tema planteado, los cuales se detallan a continuación:

Levy (2012) en su investigación nos habla, que las teorías plantean que la relación entre la tasa de interés y el crecimiento económico es directa, otros manifiestan que es inversa sobre la inversión y la producción, esto ha originado diferentes políticas monetarias, consignadas a cambiar el entorno económico y el tamaño de mercado internacional, por otro lado, manifiesta que los países desarrollados, utilizan la tasa de interés para equilibrar el tipo de cambio y controlar la inflación. En la teoría neoclásica establece la relación entre la tasa de interés y la 
inversión es inversa y directa con el ahorro; concluyendo que la teoría económica no posee una explicación con respecto a la función de la tasa de interés en el crecimiento económico.

Por otro lado, Gonzales (2015) en su trabajo de investigación sobre las alteraciones de las tasas de interés por crisis financieras y sus efectos en la economía real, establece como objetivo principal evidenciar los efectos de la volatilidad mediante el modelo VAR (vectoriales autorregresivos) y aplica el modelo de cointegración para explicar el comportamiento de largo plazo entre las variables. Al aplicar la prueba Dickey Fuller evidencia que la inversión, la tasa de interés y el consumo muestran una conducta estacionaria, concluyendo que la volatilidad de la tasa de interés se incrementa y la inversión disminuye.

Para Muller y Perrotini (2018) analizan en su artículo la intervención de la Reserva Federal en el manejo de la tasa de interés a través de la base monetaria, para esto utiliza el método econométrico tomando como evidencia la economía real de Estados Unidos y establecer su relación con los bancos centrales y el mercado internacional, llegando a la conclusión que los bancos centrales ya no usan agregados monetarios para manejar la política monetaria, utilizando la tasa nominal como instrumento para estabilizar los precios y controlar la meta de inflación.

Entre las investigaciones a nivel nacional tenemos la realizada por:

Nuñez (2016) en la que estudia la incidencia de los cambios económicos mundiales, entorno al aumento de los precios de las materias primas y el crecimiento deficiente de las economías europeas y China influyendo en la tasa de inflación, la volatilidad cambiaria, la inversión privada y pública, tomando como base la regla de Taylor para determinar que variables influyen en la actividad de la política económica. El resultado que obtuvieron muestra que las políticas utilizadas por los bancos centrales es un reflejo de la variación de las tasas de interés ante los cambios de la inflación y el tipo de cambio, llegando a la conclusión que la política monetaria conduce a la estabilidad del ciclo económico por los efectos de la inflación sobre la tasa de interés.

Las bases teóricas para la realización de esta investigación son la política monetaria, la cual analiza los estados financieros de cada sector de la economía para consolidar el sistema financiero en los que participa el sector privado y el público, compuesto por las reservas internacionales netas en moneda extranjera, el crédito interno está compuesto por el circulante y el encaje. La política monetaria depende de las características principales de cada banco central tomándose en cuenta el desarrollo en el mercado financiero de cada economía. Por otro lado, equilibrio en el mercado monetario está dado por la intersección de la demanda y oferta de dinero se obtiene la tasa de interés nominal el cual es un indicador importante para el inversionista al obtener un portafolio equilibrado se sienta satisfecho con el dinero que cuenta.

Las tasas de interés que prestan los bancos centrales son overnight, es decir, sólo un día esto es con la finalidad de cubrirlas operaciones regulares, y si los bancos deciden incrementar lo deben hacer es adquirir títulos para obtener liquidez

La política monetaria toma en cuenta dos categorías para establecer la tasa de interés: 


\section{Figura 1}

\section{Modelos de política monetaria}

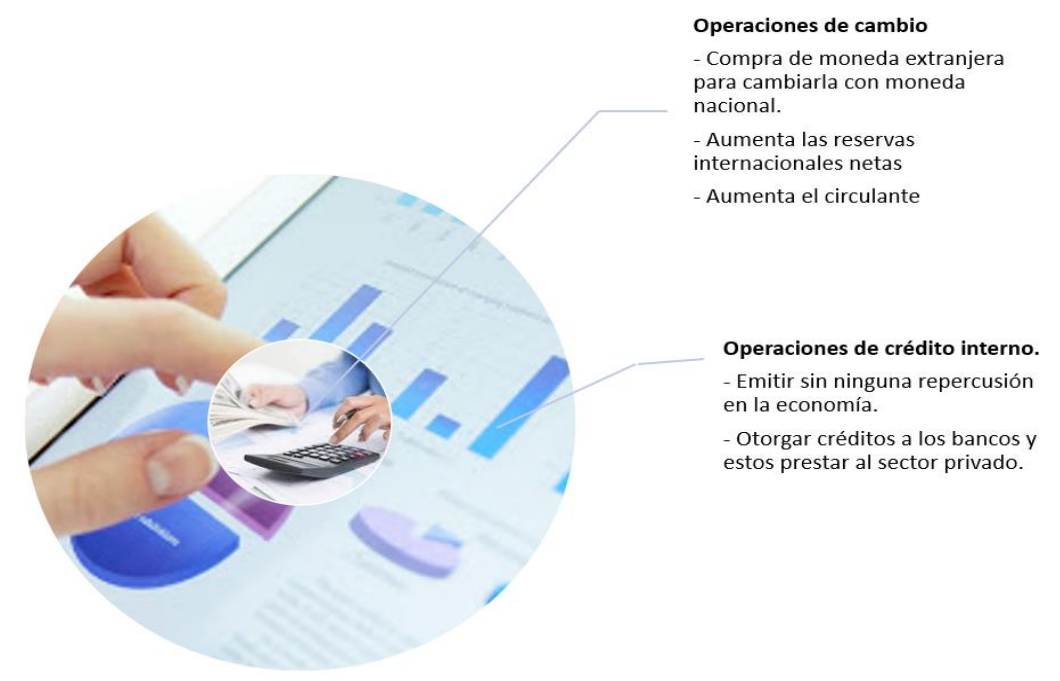

En las economías industrializadas las tasas de interés que fijan los bancos centrales están dadas por el mercado usándolas como instrumento de política monetaria, las tasas de corto plazo influyen en las operaciones menores a un año, para los de largo plazo está en función de la economía real (De Gregorio, 2007).

La otra teoría es la que tiene como base el modelo Keynesiano que establece que el ahorro es igual a la inversión en el mercado de bienes, dependiendo el ahorro del ingreso y la inversión de la tasa de interés, Keynes manifiesta que la inversión está determinada por el ingreso y la tasa de interés, donde si aumenta el ingreso la inversión aumenta una fracción en cambio si la tasa de interés aumenta la inversión disminuye, cuya fórmula es:

$$
\mathrm{I}=\Delta(\mathrm{r}, \quad \mathrm{Y}) \Delta \mathrm{I}=\mathrm{Y}(\mathrm{r})
$$

Está fórmula es la tabla de demanda de inversiones, organizada de tal manera que muestra las posibles inversiones ordenadas de mayor a menor tasa de ganancia potencial, en que las empresas deben pagar para obtener dinero y realizar la inversión (Gómez, 2011)

\section{Metodología}

El tipo de investigación es aplicada, con la finalidad de conocer procesos de la realidad para dar posibles soluciones en problemas concretos. El diseño es no experimental, se trabaja con hechos que se dieron en el entorno para encontrar también posibles soluciones al problema de estudio.

La técnica aplicada en esta investigación es el análisis de contenido cuantitativo, el cual permite recolectar los datos de manera sistemática y objetiva para clasificarlos en categorías y 
subcategorías sometiéndolo después al análisis estadístico (Fernández, 2018). La validez y la confiabilidad no se aplican porque los datos estadísticos obtenidos serán de las instituciones que generan la información.

Para el análisis de datos se usará el programa estadístico econométrico E-views que permite la estimación y resolución de modelos económicos mediante una amplia gama de procedimientos, aplicando el modelo de regresión simple para el resultado del estudio.

\section{Resultados}

El modelo econométrico, permitió determinar en qué medida incide la tasa de interés de referencia de Estados Unidos en las inversiones en la economía peruana, empleándose los siguientes indicadores para cada variable:

Para la variable tasa de interés de referencia se toma: Primero fue la tasa de interés activa para los años de 2016 al 2019 de forma trimestral, el cuál varia de $0.3 \%$ a $2.5 \%$ siendo esta los beneficios que recibe la entidad financiera al prestar el dinero.

\section{Tabla 1}

La tasa de interés activa

\begin{tabular}{|c|c|c|c|}
\hline \multicolumn{3}{|c|}{ Periodo } & \multirow{2}{*}{$\frac{\text { Tasa de interés }(\boldsymbol{\%})}{0.3}$} \\
\hline$\stackrel{\mathscr{E}}{\Xi}$ & 2016 & I & \\
\hline$\overline{\tilde{g}}$ & & II & 0.3 \\
\hline$\Xi$ & & III & 0.3 \\
\hline 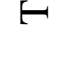 & & IV & 0.5 \\
\hline \multicolumn{4}{|c|}{2017} \\
\hline$\Xi$ & & I & 0.5 \\
\hline 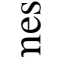 & & II & 0.5 \\
\hline 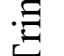 & & III & 0.5 \\
\hline$F$ & & IV & 0.8 \\
\hline \multicolumn{4}{|c|}{2018} \\
\hline$\Xi$ & & I & 1.0 \\
\hline $\bar{d}$ & & II & 1.3 \\
\hline$\Xi$ & & III & 1.3 \\
\hline & & IV & 1.5 \\
\hline \multicolumn{4}{|c|}{2019} \\
\hline$\stackrel{\Xi}{\Xi}$ & & I & 1.8 \\
\hline dै & & II & 2.0 \\
\hline$\Xi$ & & III & 2.3 \\
\hline 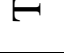 & & IV & 2.5 \\
\hline
\end{tabular}

Nota: Tasa de interés activo cargado en diferentes periodos

El segundo indicador son las operaciones de mercado abierto realizadas por el banco central de reserva para planificar adecuadamente de la política monetaria acompañados de ajuste de la tasa de interés, tipo de cambio y tasa de encaje bancario, este varia en el periodo de 2016 a 
2019 de 15,000 a 21,000 billones de dólares que permite ampliar o restringir la oferta monetaria.

\section{Tabla 2}

Operaciones de mercado abierto

\begin{tabular}{|c|c|c|c|}
\hline \multicolumn{2}{|c|}{ Periodo } & \multirow{2}{*}{$\frac{\text { OMA billones de } \$}{15,599.4}$} & \multirow{2}{*}{$\frac{\text { OMA millones de } \$}{15,599,400,000}$} \\
\hline$\Xi$ & 2016 I & & \\
\hline $\bar{\alpha}$ & II & $17,154.8$ & $17,154,800,000$ \\
\hline$\Xi$ & III & $18,658.8$ & $18,658,800,000$ \\
\hline & IV & $17,691.7$ & $17,691,700,000$ \\
\hline \multicolumn{4}{|c|}{2017} \\
\hline$\Xi$ & I & $17,963.2$ & $17,963,200,000$ \\
\hline 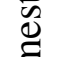 & II & $18,294.5$ & $18,294,500,000$ \\
\hline$\Xi$ & III & $18,469.5$ & $18,469,500,000$ \\
\hline 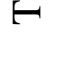 & IV & $18,769.2$ & $18,769,200,000$ \\
\hline \multicolumn{4}{|c|}{2018} \\
\hline$\Xi$ & I & $19,090.2$ & $19,090,200,000$ \\
\hline 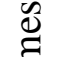 & II & 19.535 .0 & $19,535,000,000$ \\
\hline$\Xi$ & III & $19,799.5$ & $19,799,500,000$ \\
\hline$=$ & IV & $20,128.2$ & $20,128,200,000$ \\
\hline \multicolumn{4}{|c|}{2019} \\
\hline$\Xi$ & I & $20,458.5$ & $20,458,500,000$ \\
\hline$\overline{\tilde{Q}}$ & II & $20,866.8$ & $20,866,800,000$ \\
\hline$\Xi$ & III & $21,178.7$ & $21,178,700,000$ \\
\hline & IV & $21,490.1$ & $21,490,100,000$ \\
\hline
\end{tabular}

Nota: Las operaciones de mercado abierto se usa para controlar los tipos de interés.

El tercer indicador son los precios de las operaciones crediticias, conformado por el costo financiero de utilizar los recursos bancarios, para su estimación se tomó como base el prime rate (Tasa prima) usada por los bancos de Estados Unidos para operaciones consideradas de bajo riesgo con altas posibilidades de recuperación de crédito, el cual varía de acuerdo con la oferta de crédito y demanda de financiamiento. Influye en la tasa de interés y la economía. Para el periodo del 2016 al 2019 la tasa va de $3.25 \%$ a $5.50 \%$

\section{Tabla 3}

Precios de las operaciones crediticias

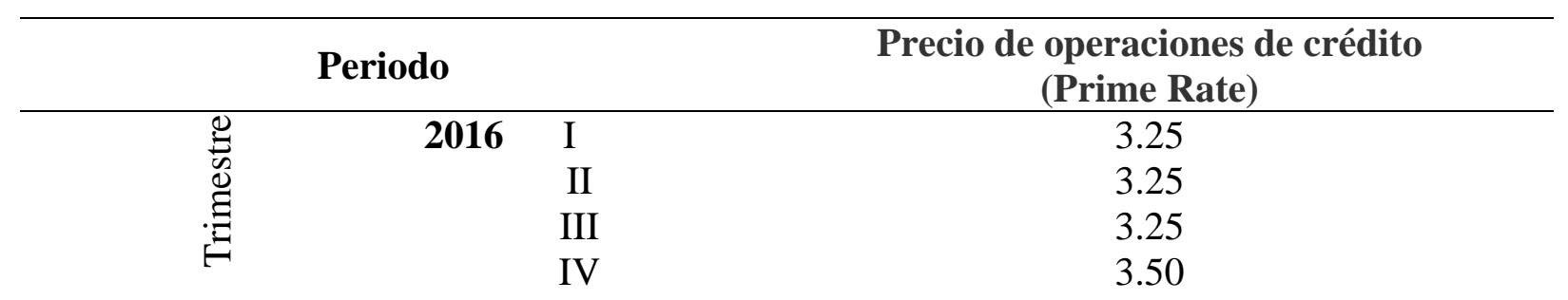




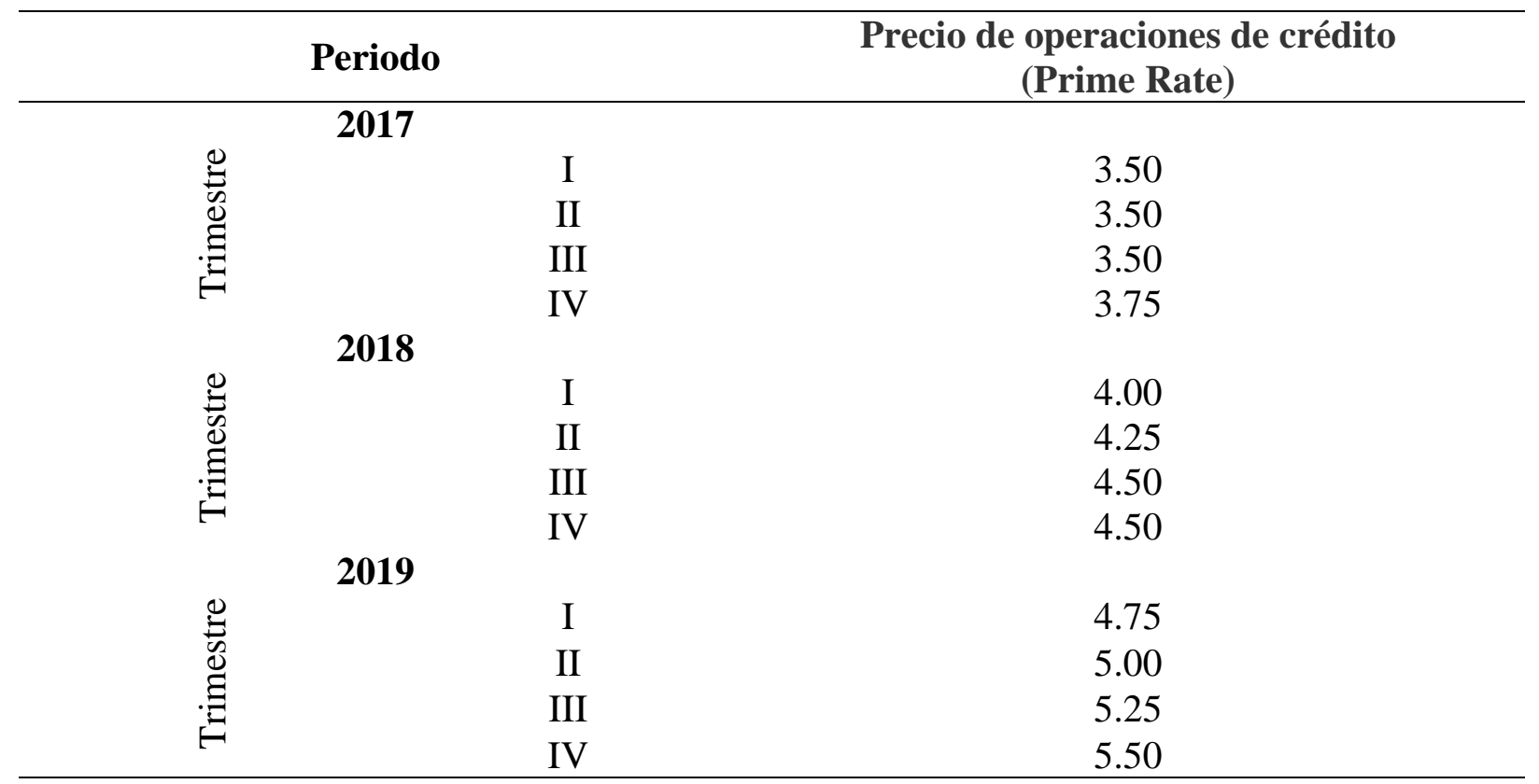

Nota: El prime rate es la tasa de fondos federales que establece la Reserva Federal.

Para calcular la inversión, el primer indicador fue el nivel de demanda agregada, el cual se refiere al flujo circular de la economía inherente a la oferta y demanda representando a todos los bienes y servicios que compran los consumidores públicos y privados, depende especialmente por la política fiscal y monetaria, originando esta última un cambio en la demanda a través de la oferta monetaria, un aumento o disminución de la tasa de interés. El nivel se toma del porcentaje del producto bruto interno siendo uno de los componentes la inversión es por eso por lo que para el periodo del 2016 al 2019 va de $2.2 \%$ a $4.4 \%$ fluctuando trimestralmente.

\section{Tabla 4}

Nivel de demanda agregada

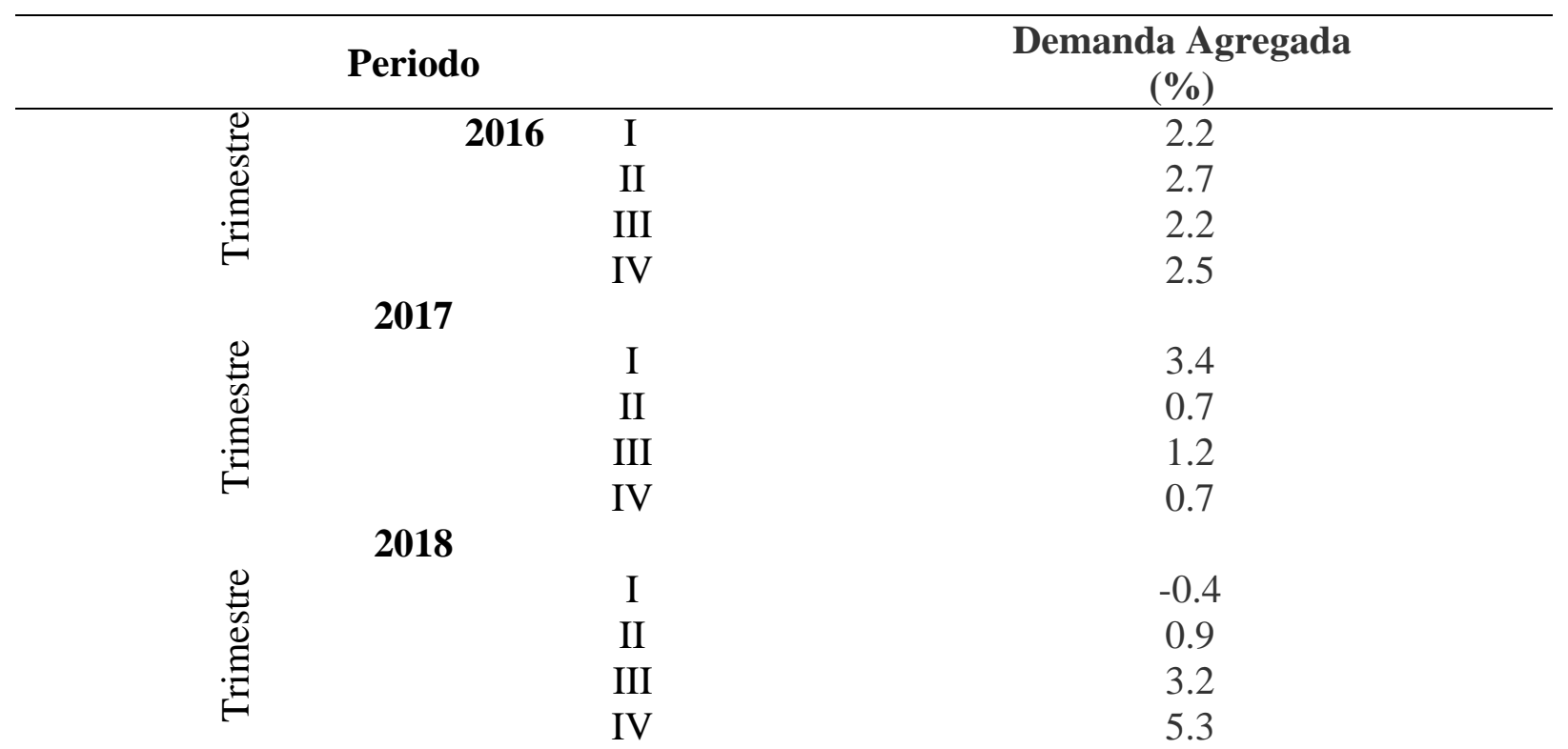




\begin{tabular}{cccc}
\hline & Periodo & & $\begin{array}{c}\text { Demanda Agregada } \\
(\%)\end{array}$ \\
\hline \multirow{\Xi}{*}{} & $\mathbf{2 0 1 9}$ & & 3.8 \\
$:$ & & I & 5.9 \\
$:$ & & II & 2.1 \\
& & III & 4.4 \\
\hline
\end{tabular}

Nota: El nivel de demanda agregada depende de la política monetaria.

El segundo indicador es el nivel de riesgo, mide las probabilidades de eventos adversos que suceden en una inversión y las consecuencias financieras que ocasiona para estimarlo se usa el riesgo país a través del EMBI PERÚ calculado por JP Morgan, se obtiene con la diferencia entre la tasa de rendimiento de los bonos de los países emergentes y la tasa del bono del tesoro estadounidense. En el periodo investigado el indicador se encuentra entre 181 puntos básicos en el 2016 y 144 puntos básicos en el 2019.

\section{Tabla 5}

Nivel de riesgo

\begin{tabular}{|c|c|c|c|}
\hline \multicolumn{2}{|c|}{ Periodo } & $\begin{array}{c}\text { EMBI PERÚ } \\
\text { (puntos básicos) }\end{array}$ & $\begin{array}{c}\text { EMBI PERÚ } \\
(\%)\end{array}$ \\
\hline \multicolumn{2}{|c|}{2016} & & \\
\hline \multirow{4}{*}{ 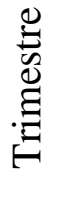 } & I & 181 & 1.81 \\
\hline & II & 168 & 1.68 \\
\hline & III & 206 & 2.06 \\
\hline & IV & 213 & 2.13 \\
\hline \multicolumn{2}{|c|}{2017} & & \\
\hline \multirow{4}{*}{ 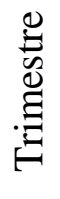 } & I & 242 & 2.42 \\
\hline & II & 206 & 2.06 \\
\hline & III & 166 & 1.66 \\
\hline & IV & 150 & 1.5 \\
\hline \multicolumn{4}{|c|}{2018} \\
\hline \multirow{4}{*}{ 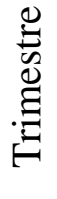 } & I & 143 & 1.43 \\
\hline & II & 140 & 1.4 \\
\hline & III & 143 & 1.43 \\
\hline & IV & 132 & 1.32 \\
\hline \multicolumn{4}{|c|}{2019} \\
\hline \multirow{4}{*}{ 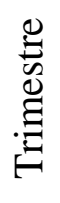 } & I & 126 & 1.26 \\
\hline & II & 163 & 1.63 \\
\hline & III & 140 & 1.4 \\
\hline & IV & 144 & 1.44 \\
\hline
\end{tabular}

Nota: Nivel de riesgo para medirla en la inversión.

El tercer indicador es el nivel de rentabilidad, para calcularlo se toma como base la tasa 
social de descuento, que indica el coste - beneficio para implementar obras públicas a través de la inversión que producirá rendimientos equilibrando el consumo actual y los beneficios futuros, estimando el desplazamiento del consumo privado cuando se da el aumento de la inversión pública exigiendo una rentabilidad mínima a los proyectos de inversión igualando la tasa de preferencia por el tiempo y por la variación de la utilidad marginal. En el periodo de investigación la tasa social de descuento se encuentra entre $8 \%$ y $11 \%$.

\section{Tabla 6}

Nivel de rentabilidad

\begin{tabular}{|c|c|c|c|}
\hline \multicolumn{3}{|c|}{ Periodo } & $\begin{array}{c}\text { Nivel de rentabilidad } \\
\text { (Tasa social de descuento \%) }\end{array}$ \\
\hline \multirow{4}{*}{ 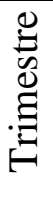 } & \multirow[t]{4}{*}{2016} & I & 8 \\
\hline & & II & 8 \\
\hline & & III & 8 \\
\hline & & IV & 8 \\
\hline \multirow{5}{*}{ 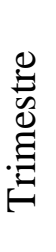 } & \multicolumn{3}{|l|}{2017} \\
\hline & & $\mathrm{I}$ & 8 \\
\hline & & II & 8 \\
\hline & & III & 9 \\
\hline & & IV & 9 \\
\hline \multicolumn{4}{|c|}{2018} \\
\hline \multirow{4}{*}{ 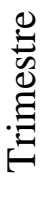 } & & $\mathrm{I}$ & 9 \\
\hline & & II & 8 \\
\hline & & III & 9 \\
\hline & & IV & 10 \\
\hline \multicolumn{4}{|c|}{2019} \\
\hline \multirow{4}{*}{ 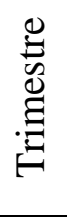 } & & I & 10 \\
\hline & & II & 11 \\
\hline & & III & 11 \\
\hline & & IV & 11 \\
\hline
\end{tabular}

Nota: La tasa social de descuento es uno de los elementos críticos en la evacuación de proyectos públicos

Después de analizar cada indicador se procedió a estimar el modelo econométrico a través del método de regresión lineal simple de acuerdo con la siguiente ecuación:

$y=\beta_{1}+\beta_{2} x_{2}+\mu$

Donde:

$\mathrm{y}=$ regresando

$\mathrm{x}=$ regresor

$\beta=$ parámetro, coeficiente, son fijos y desconocidos

$\mu=$ perturbación aleatoria o error 
De acuerdo con lo planteado se tuvo los siguientes resultados.

\section{Primera hipótesis específica:}

\section{La tasa de interés activa incide en el nivel de demanda agregada}

Para hallar la incidencia de la tasa de interés activa sobre el nivel demanda agregada ante un cambio de ésta, se aplica el modelo de regresión simple en la siguiente ecuación:

nda $=\beta_{1}+\beta_{2} * \mathrm{ti}+\mu$

Donde:

nda $=$ nivel de demanda agregada

$\mathrm{ti}=$ tasa de interés activa

$\beta=$ coeficiente de regresión

$\mu=$ perturbación aleatoria o error

Se emplea el programa econométrico E-views para estimar la ecuación obteniéndose el siguiente resultado:

\section{Tabla 7}

Método de regresión lineal simple

\begin{tabular}{lll}
\hline Variable & Coeficiente & Prob. \\
\hline $\mathbf{C}$ & 0.013214 & 0.0797 \\
$\mathbf{T i}$ & 0.011646 & 0.0501 \\
$\mathbf{R}_{\mathbf{2}}$ & 0.247085 & \\
\hline
\end{tabular}

Fuente: elaboración propia

Según el $\mathrm{R}_{2}(0.247085)$ indica que ante una variación de la tasa de interés activa explica el $24 \%$ del nivel de la demanda agregada.

Para hallar la incidencia se partirá de los siguientes supuestos:

Primero: La tasa de interés activa es de $2.5 \%$, reemplazando en la ecuación se tiene: $\mathrm{nda}=0.013214+0.011646 * \mathrm{ti}$ nda $=0.013214+0.011646 * 0.025$

nda $=0.01350515$

$\mathrm{nda}=1.35 \%$

Esto significa que la tasa de interés activa incide en $1.35 \%$ el nivel de demanda agregada.

Segundo: La tasa de interés activa se incrementa a 3.25\%, reemplazando la ecuación se tiene: $\mathrm{nda}=0.013214+0.011646 * \mathrm{ti}$ $\mathrm{nda}=0.013214+0.011646 * 0.035$ 
nda $=0.01362161$

$\mathrm{nda}=1.36 \%$

Esto significa que si la tasa de interés activa se incrementa en $1 \%$ incide en $1.36 \%$ el nivel de demanda agregada.

Tercero: La tasa de interés activa se reduce a 1.25\%, reemplazando la ecuación se tiene:

nda $=0.013214+0.011646 * \mathrm{ti}$

nda $=0.013214+0.011646 * 0.0125$

nda $=0.01338869$

nda $=1.34 \%$

Esto significa que si la tasa de interés activa se reduce en $1 \%$ incide en $1.34 \%$ el nivel de demanda agregada.

De acuerdo con los resultados obtenidos, cuando la tasa de interés sube en $1 \%$ el nivel de demanda agregada varía en $0.86 \%$ y cuando baja en $1 \%$ varía en $0.87 \%$

Con la finalidad de verificar la viabilidad del modelo se comprobó la autocorrelación y la heterocedasticidad (tabla 8)

\section{Tabla 8}

Test de comprobación

\begin{tabular}{lllll}
\hline Autocorrelación & DW & 2.00 & & \\
Heterocedasticidad & F-statistic & 2.381431 & Prob. F (2,3) & 0.1315 \\
& Obs*R-squared & 4.290175 & Prob. Chi- square (2) & 0.1171 \\
& Scaled explained ss & 1.916343 & Prob. Chi- square (2) & 0.3836 \\
\hline
\end{tabular}

Fuente: elaboración propia

Se aplicó el estadístico d de Durbin-Watson, cuyo valor es 2 por lo que significa que el modelo no tiene autocorrelación, para verificar que no tiene heterocedasticidad se realizó el contraste de White siendo la prob. F (0.1315) mayor a 0.05 el cual nos dice que las varianzas de los errores se distribuyen constantemente en el periodo estudiado.

\section{Segunda hipótesis específica:}

\section{Las operaciones de mercado abierto inciden en el nivel de riesgo}

Para hallar la incidencia de las operaciones de mercado abierto sobre el nivel de riesgo ante un cambio de estas, se aplica el modelo de regresión simple en la siguiente ecuación:

nrie $=\beta_{1}+\beta_{2} *$ oma $+\mu$

Donde: 
nrie $=$ nivel de riesgo.

oma $=$ operaciones de mercado abierto

$\beta=$ coeficiente de regresión

$\mu=$ perturbación aleatoria o error

Se emplea el programa econométrico E-views para estimar la ecuación obteniéndose el siguiente resultado:

\section{Tabla 9}

Método de regresión lineal simple

\begin{tabular}{lll}
\hline Variable & Coeficiente & Prob. \\
\hline C & 412.1886 & 0.0004 \\
OMA & $-1.29 \mathrm{E}-08$ & 0.0143 \\
$\mathbf{R}_{\mathbf{2}}$ & 0.35 & \\
\hline
\end{tabular}

Fuente: elaboración propia.

Según el $\mathrm{R}_{2}(0.35)$ indica que ante una variación de las operaciones de mercado abierto explica el $35 \%$ al nivel de riesgo.

Para hallar la incidencia se partió de los siguientes supuestos:

Primero: Las operaciones de mercado abierto son de USD\$21,490¹00,000 millones de dólares, reemplazando en la ecuación se tiene:

nrie $=412.1886-1.29 \mathrm{E}-08 *$ oma

nrie $=412.1886-1.29 \mathrm{E}-08^{*} 21,490^{\prime} 100,000$

nrie $=134.97$

nrie $=134.97 / 100=1.35 \%$ $1.35 \%$.

Esto significa que las operaciones de mercado abierto inciden en el nivel de riesgo en

Segundo: Las operaciones de mercado abierto son de USD\$21,590`100,000 millones de dólares, reemplazando en la ecuación se tiene:

nrie $=412.1886-1.29 \mathrm{E}-08 *$ oma

nrie $=412.1886-1.29 \mathrm{E}-08^{*} 21,590^{\prime} 100,000$

nrie $=133.68$ puntos básicos

nrie $=133.68 / 100=1.34 \%$

Esto significa que las operaciones de mercado abierto inciden en el nivel de riesgo en $1.34 \%$ cuando se incrementan en USD $\$ 100 ’ 000,000$

Tercero: Las operaciones de mercado abierto son de USD\$21,390`100,000 millones de dólares, reemplazando en la ecuación se tiene: 
nrie $=412.1886-1.29 \mathrm{E}-08 *$ oma

nrie $=412.1886-1.29 \mathrm{E}-08^{*} * 21,390^{\prime} 100,000$

nrie $=136.26$ puntos básicos

nrie $=136.26 / 100=1.36 \%$

Esto significa que las operaciones de mercado abierto inciden en el nivel de riesgo en $1.36 \%$ cuando se reducen en USD $\$ 100 ` 000,000$.

De acuerdo con los resultados obtenidos, cuando las operaciones de mercado abierto se incrementan en USD\$100’000,000 millones de dólares el nivel de riesgo varía en $0.96 \%$ y cuando disminuye el nivel de riesgo varía en $0.95 \%$.

Con la finalidad de verificar la viabilidad del modelo se comprobó la autocorrelación y la heterocedasticidad (tabla 10)

\section{Tabla 10}

Test de comprobación

\begin{tabular}{|c|c|c|c|c|}
\hline Autocorrelación & DW & 2.00 & & \\
\hline \multirow[t]{3}{*}{ Heterocedasticidad } & F-statistic & 1.594085 & Prob. F $(2,3)$ & 0.2404 \\
\hline & Obs*R-squared & 3.151111 & $\begin{array}{l}\text { Prob. Chi- } \\
\text { square (2) }\end{array}$ & 0.2069 \\
\hline & $\begin{array}{l}\text { Scaled explained } \\
\text { ss }\end{array}$ & 1.810736 & $\begin{array}{l}\text { Prob. Chi- } \\
\text { square (2) }\end{array}$ & 0.4044 \\
\hline
\end{tabular}

Fuente: elaboración propia

Se aplicó el estadístico d de Durbin-Watson, cuyo valor es 2 por lo que significa que el modelo no tiene autocorrelación, para verificar que no tiene heterocedasticidad se realizó el contraste de White siendo la prob. F (0.2404) mayor a 0.05 el cual nos dice que las varianzas de los errores se distribuyen constantemente en el periodo estudiado.

\section{Tercera hipótesis específica}

\section{El precio de las operaciones crediticias incide en el nivel de rentabilidad}

Para hallar el precio de las operaciones crediticias sobre el nivel de rentabilidad ante un cambio de estas, se aplica el modelo de regresión simple en la siguiente ecuación:

nren $=\beta_{1}+\beta_{2} * \mathrm{pr}+\mu$

Donde:

nren $=$ nivel de rentabilidad .

$\mathrm{pr}=$ precio de las operaciones crediticias

$\beta=$ coeficiente de regresión

$\mu=$ perturbación aleatoria o error 
Se emplea el programa econométrico E-views para estimar la ecuación obteniéndose el siguiente resultado:

\section{Tabla 11}

Método de regresión lineal simple

\begin{tabular}{lll}
\hline Variable & Coeficiente & Prob. \\
\hline $\mathbf{C}$ & 0.032838 & 0.0005 \\
$\mathbf{P R}$ & $1.42 \mathrm{E}-02$ & 0.0000 \\
$\mathbf{R}_{\mathbf{2}}$ & 0.823768 & \\
\hline
\end{tabular}

Fuente: elaboración propia

Según el $\mathrm{R}_{2}(0.82)$ indica que ante una variación del precio de las operaciones crediticias (prima rate) explica el $82 \%$ al nivel de rentabilidad.

Para hallar la incidencia se partirá de los siguientes supuestos:

Primero: El precio de las operaciones crediticias es de 5.5\%, reemplazando en la ecuación se tiene:

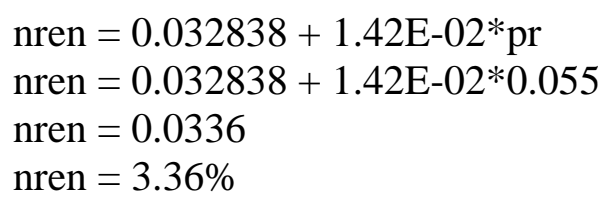

Esto significa que si el precio de las operaciones crediticias es 5,5\% inciden en el nivel de rentabilidad en $3.36 \%$

Segundo: El precio de las operaciones crediticias se incrementa a 6.5\%, reemplazando en la ecuación se tiene:

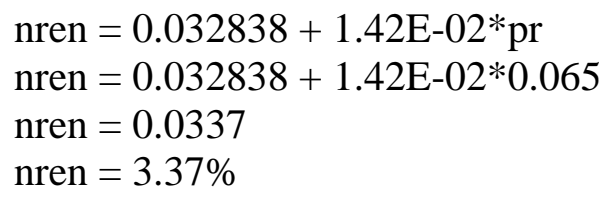

Esto significa que si el precio de las operaciones crediticias se incrementa a $6,5 \%$ inciden en el nivel de rentabilidad en $3.37 \%$

Tercero: El precio de las operaciones crediticias se reducen a 4.5\%, reemplazando en la ecuación se tiene:

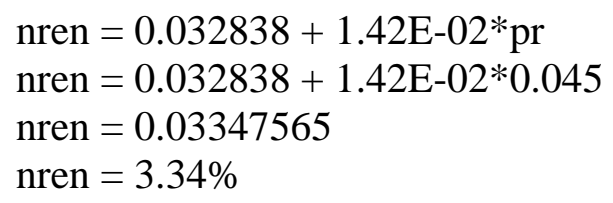


Esto significa que si el precio de las operaciones crediticias se reduce a 4,5\% incide en el nivel de rentabilidad en $3.34 \%$

De acuerdo con los resultados obtenidos, cuando el precio de las operaciones crediticias (prima rate) se incrementa en $1 \%$ el nivel de rentabilidad varia en $0.47 \%$ y cuando disminuye el nivel de rentabilidad varía en $0.59 \%$

Con la finalidad de verificar la viabilidad del modelo se comprobó la autocorrelación y la heterocedasticidad (tabla 12)

\section{Tabla 12}

Test de comprobación

\begin{tabular}{lllll}
\hline Autocorrelación & DW & 2.32605 & & \\
Heterocedasticidad & F-statistic & 1.602308 & Prob. F (2,3) & 0.2388 \\
& Obs*R-squared & 3.164151 & Prob. Chi- square (2) & 0.2055 \\
& Scaled explained ss & 3.974034 & Prob. Chi- square (2) & 0.1371 \\
\hline
\end{tabular}

Fuente: elaboración propia

Se aplicó el estadístico d de Durbin-Watson, cuyo valor es 2.32605 por lo que significa que el modelo no tiene autocorrelación, para verificar que no tiene heterocedasticidad se realizó el contraste de White siendo la prob. F (0.2388) mayor a 0.05 el cual nos dice que las varianzas de los errores se distribuyen constantemente en el periodo estudiado.

\section{Hipótesis General}

\section{La tasa de interés de referencia de Estados Unidos incide en las inversiones en la economía peruana}

Para hallar la incidencia de la tasa de interés de referencia sobre las inversiones en la economía peruana ante un cambio de esta, se aplica el modelo de regresión simple en la siguiente ecuación:

$\operatorname{inv}=\beta_{1}+\beta_{2} * \mathrm{ti}+\mu$

Donde:

inv = inversión

ti $=$ tasa de interés de referencia

$\beta=$ coeficiente de regresión

$\mu=$ perturbación aleatoria o error

Se emplea el programa econométrico E-views para estimar la ecuación obteniéndose el siguiente resultado: 


\section{Tabla 13}

Método de regresión lineal simple

\begin{tabular}{lll}
\hline Variable & Coeficiente & Prob. \\
\hline $\mathbf{C}$ & 3.608148 & 0.0000 \\
$\mathbf{T i}$ & 0.757037 & 0.0035 \\
$\mathbf{R}_{\mathbf{2}}$ & 0.46777 & \\
\hline
\end{tabular}

Fuente: elaboración propia

Según el $\mathrm{R}_{2}(0.46777)$ indica que ante una variación de la tasa de interés de referencia explica el $47 \%$ de la inversión en la economía peruana.

Para hallar la incidencia se partirá de los siguientes supuestos:

Primero: La tasa de interés de referencia es de $2.5 \%$, reemplazando en la ecuación se tiene: inv $=3.608148+0.757037 * \mathrm{ti}$

inv $=3.608148+0.757037 * 0.025$

inv $=3.62707393$

inv $=36 \%$ $36 \%$

Esto significa que si la tasa de interés de referencia es de $2.5 \%$ incide en la inversión en

Segundo: La tasa de interés de referencia se incrementa a 3.5\%, reemplazando en la ecuación se tiene:

inv $=3.608148+0.757037 * \mathrm{ti}$

inv $=3.608148+0.757037 * 0.035$

inv $=3.6346443$

inv $=36 \%$

Esto significa que si la tasa de interés de referencia se incrementa a 3.5\% incide en la inversión en $36 \%$

Tercero: La tasa de interés de referencia se reduce a 1.5\%, reemplazando en la ecuación se tiene:

inv $=3.608148+0.757037 * \mathrm{ti}$

inv $=3.608148+0.757037 * 0.015$

inv $=3.61950356$

inv $=36 \%$

Esto significa que si la tasa de interés de referencia se reduce a $1.5 \%$ incide en la inversión en $36 \%$.

De acuerdo con los resultados obtenidos, cuando la tasa de interés de referencia se 
incrementa o disminuya en $1 \%$ incide en la inversión en $36 \%$.

Con la finalidad de verificar la viabilidad del modelo se comprobó la autocorrelación y la heterocedasticidad (tabla 8)

\section{Tabla 14}

Test de comprobación

\begin{tabular}{lllll}
\hline Autocorrelación & DW & 2.00 & & \\
Heterocedasticidad & F-statistic & 2.736047 & Prob. F (2,3) & 0.1019 \\
& Obs*R-squared & 4.739771 & Prob. Chi- square (2) & 0.0935 \\
& Scaled explained ss & 3.023224 & Prob. Chi- square (2) & 0.2206 \\
\hline
\end{tabular}

Fuente: elaboración propia

Se aplicó el estadístico d de Durbin-Watson, cuyo valor es 2 por lo que significa que el modelo no tiene autocorrelación, para verificar que no tiene heterocedasticidad se realizó el contraste de White siendo la prob. F (0.1019) mayor a 0.05 el cual nos dice que las varianzas de los errores se distribuyen constantemente en el periodo estudiado.

\section{Conclusiones}

De acuerdo con los resultados obtenidos se evidencia que el coeficiente R2 es 0.24 (24\%) mide la tasa de interés activa sobre el nivel de demanda agregada, lo que significa que ante una variación de $1 \%$ en la tasa de interés activa incide en $1.36 \%$ cuando sube y $1.34 \%$ cuando baja para el nivel de la demanda agregada.

De acuerdo con los resultados obtenidos se evidencia que el coeficiente R2 es 0.35 (35\%) mide las operaciones de mercado abierto sobre el nivel de riesgo, lo que significa que ante una variación de USD \$100`000,000 de dólares en las operaciones de mercado abierto incide en $1.33 \%$ cuando sube y $1.36 \%$ cuando baja para el nivel de riesgo.

De acuerdo con los resultados obtenidos se evidencia que el coeficiente R2 es 0.82 (82\%) mide el precio de las operaciones sobre el nivel de rentabilidad, lo que significa que ante una variación de $1 \%$ en el precio de las operaciones crediticias incide en $1.34 \%$ cuando sube y $1.33 \%$ cuando baja para el nivel de rentabilidad.

De acuerdo con los resultados obtenidos se evidencia que el coeficiente R2 es 0.47 (47\%) mide la tasa de interés de referencia de Estados Unidos sobre la inversión en la economía peruana, lo que significa que ante una variación de $1 \%$ en la tasa de interés de referencia, ésta incide en $36 \%$ en la inversión de la economía peruana.

\section{Referencias Bibliográficas}

Angulo, A, Barbarán, J, Sotelo, F y Zimic, O. (2017). Planeamiento Estratégico del Banco Central de Reserva del Perú. Lima, Perú: Pontificia Universidad Católica del Perú. 
Asatarita, R. (2015). Keynes sobre inversión y ahorro. Recuperado de: https://rolandoastarita.blog/2015/12/01/keynes-sobre-inversion-y-ahorro/

Banco Central de Reserva (2018). Reporte de Inflación. Recuperado de:

http://www.bcrp.gob.pe/docs/Publicaciones/Reporte-Inflacion/2018/diciembre/reporte-deinflacion-diciembre-2018.pdf

Banco Central de Reserva (2017). Importancia de la tasa de interés de referencia del BCRP. Recuperado de:

http://www.bcrp.gob.pe/docs/Publicaciones/Revista-Moneda/moneda-170/moneda-170.pdf

Banco Central de Reserva (2019). La Política Monetaria del Bcrp y la Tasa de Interés de la Fed. Recuperado de: https://www.bcrp.gob.pe/publicaciones/revista-moneda/revista-moneda-177.html

Banco Central de Reserva (2019). Hoja resumen de los indicadores económicos. Recuperado de: https://www.bcrp.gob.pe/docs/Estadisticas/NEDD/Hojas.htm

Cermeño, R, Dancourt, O, Ganiko, G y Mendoza, W (2016). Tasas de interés activas y política monetaria en el Perú. Un análisis con datos de bancos individuales. Recuperado de: http://revistas.pucp.edu.pe/index.php/economia/article/view/16515/16870 http://doi.org/10.18800/economia.201602.004

Comisión Económica para América Latina y el Caribe (2019). La Inversión Extranjera Directa en América Latina y el Caribe 2019(LC/PUB.2019/16-P). Recuperado de: https://repositorio.cepal.org/handle/11362/44697

Daza, F y Uribe, J (2016). Efectos de los cambios de la tasa de interés de Estados Unidos sobre Colombia, Perú y Chile. Recuperado de:

http://www.scielo.org.co/pdf/ecoca/n17/n17a06.pdf.

De Gregorio, J. (2012). Macroeconomía: Teoría y Políticas (1ra. ed). Recuperado de: http://www.degregorio.cl/pdf/Macroeconomia.pdf

FedprimeRate (2019). Historial de las tasas preferenciales. Recuperado de: http://www.fedprimerate.com/wall_street_journal_prime_rate_history.htm\#current

Flores, M. (2017). Efecto crowding-in entre la inversión pública y privada en el Perú - análisis de los efectos contemporáneos y futuros de la inversión del sector público sobre la inversión del sector privado (1999 - 2014). Lima, Perú: Universidad de Lima.

Instituto Nacional de Estadística e informática (2019). Principales Indicadores. Recuperado en: https://www.inei.gob.pe/estadisticas/indice-tematico/economia/

JPMorgan Chase y Co. (2019). Tasa de preferencias históricas. Recuperado de: https://about.jpmorganchase.com/about/our-business/historical-prime-rate

Gray, S. y Talbot, N. (2006). Monetary operations. Recuperado de: https://www.bcrp.gob.pe/docs/Publicaciones/Publicaciones-de-Otras-Instituciones/puben-74.pdf

Levy, N. (2012). Tasas de interés, demanda efectiva y crecimiento económico. Recuperado de: http://www.scielo.org.mx/scielo.php?script=sci_arttext\&pid=S1665-952X2012000100005

Lira, P. (2016). La Tasa de Referencia: Significado, efectividad e implicancias. Recuperado de: https://gestion.pe/blog/deregresoalobasico/2016/06/la-tasa-de-referencia-significado-efectividade-implicancias.html?ref=gesr

Ministerio de economía y finanzas (2017). Actualización de la tasa social de descuento. Recuperado en:

https://www.mef.gob.pe/contenidos/inv_publica/docs/parametros_evaluacion_social/Tasa _Social_Descuento.pdf 
Muller, N y Perrotini, I. (2018). ¿Existe alguna relación entre la base monetaria y la tasa de interés de la Reserva Federal de los Estados Unidos? Recuperado de:

http://www.cya.unam.mx/index.php/cya/article/view/1905/1289

Núñez, C. (2016). Política monetaria y política fiscal en el Perú entre 1990 y 2013. Recuperado de:

http://tesis.pucp.edu.pe/repositorio/bitstream/handle/123456789/8164/nu\%c3\%91ez_atencio_car los_emmanuel_politica.pdf?sequence $=1$

Ochoa, O. (2013). Las operaciones de mercado abierto -OMAS-Instrumento eficaz para el control de la Base monetaria: trasegar histórico y pertinencia actual. Recuperado de: https://www.revistamisionjuridica.com/las-operaciones-de-mercado-abierto-omasinstrumento-eficaz-para-el-control-de-la-base-monetaria-trasegar-historico-y-pertinenciaactual/

Proinversión (2019). Estadísticas de Inversión Extranjera. Recuperado: http://www.investinperu.pe/modulos/JER/PlantillaStandard.aspx are=0\&prf=0\&jer=5652 $\& \sec =1$

Sala de inversiones. (2018). Por qué son importantes las tasas de interés de referencia mundiales. Recuperado de:

https://www.saladeinversion.com/articulos/por-que-son-importantes-las-tasas-de-interes-dereferencia-mundiales-9276955/

The Federal Reserve Bank of New York (2018). Financial Statements. Recuperado de: https://www.newyorkfed.org/medialibrary/media/aboutthefed/annual/annual18/FinancialStatements.pdf

The Federal Reserve Board (2019). Monetary Policy.Recuperado de: https://www.federalreserve.gov/monetarypolicy.htm

The Federal Reserve Board (2019). Board of Governors of the Federal Reserve System. Recuperado:

https://www.federalreserve.gov/releases/h41/ 